\title{
Stability of stationary and time-varying nongyrotropic particle distributions
}

\author{
A. L. Brinca \\ Centro de F. de Plasmas, Instituto Superior Técnico, P-1096 Lisboa Codex, Portugal
}

Received: 2 June 1998 / Accepted 18 August 1998

\begin{abstract}
The ubiquity of nongyrotropic particle populations in space plasmas warrants the study of their characteristics, in particular their stability. The unperturbed nongyrotropic distribution functions in homogeneous media without sources and sinks (closed phase space) must be rotating and time-varying (TNG), whereas consideration of open phase spaces allows for the occurrence of homogeneous and stationary distributions (SNG). The free energy brought about by the introduction of gyrophase organization in a particle population can destabilize otherwise thoroughly stable magnetoplasmas (or, a fortiori, enhance pre-existing gyrotropic instabilities) and feed intense wave growth both in TNG and SNG environments: The nongyrotropic (electron or ion) species can originate unstable coupling among the gyrotropic characteristic waves. The stability properties of these two types of homogeneous nongyrotropy shall be contrasted for parallel (with respect to the ambient magnetic field) and perpendicular propagation, and their potential role as wave activity sources shall be illustrated resorting to solutions of the appropriate dispersion equations and numerical simulations.
\end{abstract}

Key words. Space plasma physics (waves and instabilities) - Magnetospheric physics (plasma waves and instabilities) - Interplanetary physics (plasma waves and turbulence)

\section{Introduction}

The velocity distribution in the perpendicular (with respect to the ambient magnetic field $\mathbf{B}_{o}=B_{o} \hat{\mathbf{x}}$ ) plane of

Correspondence to: ebrinca@beta.ist.utl.pt nongyrotropic particle populations depends on the gyrophase angle $\varphi=\arctan \left(v_{z} / v_{y}\right)$. The first reported space observations of particles exhibiting gyrophase organization described nongyrotropic ions in the Earth's foreshock region (Eastman et al., 1981) and several Earth radii upstream (Gurgiolo et al., 1981). Since then many other observations have confirmed the frequent occurrence of nongyrotropic populations in most regions of the geoplasma and in cometary environments (Coates et al., 1993).

The ubiquity of gyrophase bunching in space plasmas begs investigation of its origins and effects. Mechanisms capable of generating nongyrotropy include the gyrophase filtering associated with reflection and transmission at the Earth's bow shock (Burgess, 1987), nonlinear wave-particle interactions (Hoshino and Terasawa, 1985), inhomogeneous ionization (Neubauer et al., 1993), magnetized double layers (Borovski, 1988), substorm dipolarization (Delcourt et al., 1997) and inhomogeneous current sheets (Motschmann and Glaßmeier, 1997). The consequences of nongyrotropy, first studied by Sudan (1965), are the subject of current intense research (e.g. Cao et al., 1998; Motschmann and Glaßmeier, 1998; Brinca and Romeiras, 1998) and should thus remain in the forseeable future.

A brief review of previous theoretical investigations on the stability of homogeneous (time-varying and stationary) nongyrotropic particle distributions is provided below when the dispersion equations for parallel and perpendicular propagation are addressed. Here we shall be concerned with the stability of homogeneous time-varying nongyrotropic distributions (TNG) and stationary nongyrotropic distributions (SNG) magnetoplasmas with respect to parallel and perpendicular wave propagation. The underlying studies have adopted analytic or simulation approaches and the given illustrations shall provide examples of both. In a nutshell, introduction of nongyrotropy in a magnetoplasma brings about coupling among the eigenmodes of gyrotropic propagation that can destabilize an otherwise thouroughly stable medium or, all the more, enhance 
previously existing gyrotropic instabilities. The free energy associated with gyrophase organization can thus feed distinct types of wave activity.

\section{Unperturbed nongyrotropic distributions}

Adopting cylindrical coordinates in velocity space, the unperturbed (zero wave fields) distribution function of a nongyrotropic particle population identified by the subscript $b, F_{b o}\left(v_{\perp}, v_{x}, \mathbf{r}, t\right)$, in a collisionless magnetoplasma without source $(S)$ and loss $(L)$ terms (closed phase space) satisfies the Vlasov equation,

$\left(\frac{\partial}{\partial t}+\mathbf{v} \cdot \frac{\partial}{\partial \mathbf{r}}-\Omega_{b} \frac{\partial}{\partial \varphi}\right) F_{b o}=0$

where $\Omega_{b}$ stands for the signed angular cyclotron frequency of the nongyrotropic species.

Restricting attention to homogeneous media, as is the case here, it becomes clear that nongyrotropic unperturbed distributions (depending on $\varphi$ ) in closed phase spaces have to be time-varying rotating, $F_{b o}=$ $F_{b o}\left(v_{\perp}, v_{x}, \varphi+\Omega_{b} t\right)$. We shall use the acronym TNG to identify these distributions.

Consideration of open phase spaces (finite source and, or, loss terms) leads to the inclusion of $S_{o}-L_{o}$ on the rhs of the above (transport) equation (the subscript $o$ identifies unperturbed terms). Now $F_{b o}$ can be both homogeneous in space and constant in time: $F_{b o}\left(v_{\perp}, v_{x}, \varphi\right)$. We refer to these solutions as $\mathrm{SNG}$ distributions, recognizing that other possibilities may occur (viz. also homogeneous and time-varying nongyrotropies); specific examples can be found in the literature (e.g. Motschmann et al., 1997).

As already stressed, TNG and SNG distributions do not constitute a complete set of nongyrotropic populations. For example, both in closed and open phase spaces it is possible to find inhomogeneous unperturbed distributions exhibiting gyrophase organization. The TNG and SNG models are adopted here for the sake of simplicity, with the further proviso that the gyrophase dependence is separable and (necessarily) $2 \pi$ periodic, $F_{b o}=G_{b o}\left(v_{\perp}, v_{x}\right) \Phi(\Delta)$, where $\Delta=\varphi+\Omega_{b} t(\Delta=\varphi)$ for the TNG (SNG) model, with

$$
\begin{aligned}
& \Phi(\Delta)=\sum_{n=-\infty}^{\infty} \phi_{n} \mathrm{e}^{-i n \Delta}, \quad \phi_{n}=\frac{1}{2 \pi} \int_{2 \pi} \Phi(\Delta) \mathrm{e}^{i n \Delta} \mathrm{d} \Delta, \\
& \Phi \geq 0, \quad \phi_{n}=\left(\phi_{-n}\right)^{*}, \quad \int_{2 \pi} \Phi(\Delta) \mathrm{d} \Delta=1=2 \pi \phi_{o} .
\end{aligned}
$$

Extreme examples of gyrophase organization are the monochromatic (gyrophases share a common value),

$\Phi_{b}(\Delta)=\Phi_{m}(\Delta)=\delta(\Delta), \quad \phi_{n}=\frac{1}{2 \pi}$,

or dichromatic (gyrophases are evenly distributed between two values differing by $\pi$ ),

$$
\begin{aligned}
& \Phi_{b}(\Delta)=\Phi_{d}(\Delta)=\frac{1}{2}[\delta(\Delta)+\delta(\Delta-\pi)], \\
& \phi_{n}=\frac{1}{2 \pi}(n=0, \pm 2, \pm 4, \ldots), \quad \phi_{n}=0(n= \pm 1, \pm 3, \ldots),
\end{aligned}
$$

distributions (defined in the interval $-\pi / 2 \leq \Delta<3 \pi / 2$, and $2 \pi$ periodic elsewhere). Their utilization facilitates the ensuing analysis, but the consequences of adopting more relistic distributions shall be commented upon.

\section{On the dispersion equation}

The dispersion and stability study is based on the standard linearization and (Fourier in time and Laplace in space) transformation of the Maxwell and Vlasov equations. The envisaged homogeneous magnetoplasma is neutral, parallel-current free (parallel drifts are not precluded) and, besides the nongyrotropic population, contains electrons (subscript $e$ ) and protons (subscript $p$ ) with unperturbed distribution functions $F_{o l}\left(v_{\perp}, v_{x}\right)$, $l=e, p$. The study of the SNG model (open phase spaces, in general) requires characterization of the source, $S$, and loss, $L$, terms in the Vlasov equation for the nongyrotropic species, in particular of their perturbations $S_{1}$ and $L_{1}$. If it is assumed that $S_{1}=L_{1}=0$, that is $S=S_{o}$ and $L=L_{o}$, this linearized perturbed Vlasov equation does not carry information (the same happening with the dispersion equation) on the sources and sinks of the nongyrotropic population: they yield the adopted unperturbed solution $F_{b o}=G_{b o}\left(v_{\perp}, v_{x}\right) \Phi(\varphi)$, but they are "external" to the system and do not exert further influence on its linear behavior. Unless stated otherwise, we shall discuss here the "external" SNG model.

The separate analyses of parallel and perpendicular propagation found below are not concerned with the actual derivation of the relevant wave and dispersion equations that can be found in the cited references. Rather, emphasis is placed on highlighting aspects that contribute to the understanding of mechanisms associated with the nongyrotropic effects brought about by the introduction of gyrophase organization. Specific examples of nongyrotropic dispersion and stability are provided in another section.

\subsection{Parallel propagation}

Parallel propagation in TNG magnetoplasmas was first studied by Sudan (1965); more recently, Brinca et al. (1992, 1993a, 1993b) and Motschmann and Glaßmeier (1993) pursued the analysis farther. Cao et al. (1995), Motschmann et al. (1997) and Brinca and Romeiras (1998) investigated the parallel stability of the "external" SNG model. In both media the existence of a nongyrotropic species can couple the characteristic modes of parallel propagation $(+$ and - : left- and right-hand circularly polarized transverse electromagnetic modes; $x$ : longitudinal electrostatic mode). 
Adoption of "gyrating" coordinates, ()$_{ \pm}=\left[()_{y} \pm\right.$ $\left.i()_{z}\right] / 2$, and use of the transformed Maxwell equations yields

$\left(k^{2} c^{2}-\omega^{2}\right) \bar{E}_{ \pm}=i \frac{\omega}{\epsilon_{o}} \bar{J}_{ \pm}, \quad \bar{E}_{x}=-i \frac{1}{\omega \epsilon_{o}} \bar{J}_{x}$,

evidencing that the characteristic modes can be generated by similar current densities (the bar denotes complex amplitude transformed quantities). Hence, if interaction among the eigenmodes induced by nongyrotropy occurs, gyrophase organization must be responsible for the generation of current densities not (wholly) originated in modes of identical polarization: understanding the nongyrotropic coupling is tantamount to interpreting the stimulation of the current densities.

Centering the discussion in the nongyrotropic species and using $f_{b}$ to represent the perturbation in $N_{b} F_{b}$, the transformed $\bar{f}_{b}=\bar{f}_{b}\left(\omega, k ; v_{x}, v_{\perp}, \varphi\right)$ must also be $2 \pi$ periodic in $\varphi$,

$\bar{f}_{b}=\sum_{m=-\infty}^{\infty} \bar{f}_{m b} \mathrm{e}^{-i m \varphi}$,

with $\bar{f}_{m b} \exp (-i m \varphi)$ representing in the real domain, at a fixed position, a gyrophase structure rotating with angular velocity $\mathrm{d} \varphi / \mathrm{d} t=\omega / m$.

Recalling that

$$
\overline{\mathbf{J}}_{b}=q_{b} \int_{0}^{2 \pi} \mathrm{d} \varphi \int_{-\infty}^{\infty} \mathrm{d} v_{x} \int_{0}^{\infty} \mathrm{d} v_{\perp} v_{\perp} \mathbf{v} \bar{f}_{b},
$$

we arrive at

$$
\bar{J}_{x b}=2 \pi q_{b} \int_{-\infty}^{\infty} \mathrm{d} v_{x} \int_{0}^{\infty} \mathrm{d} v_{\perp} v_{\perp} v_{x} \bar{f}_{o b}
$$

and

$\bar{J}_{ \pm b}=\pi q_{b} \int_{-\infty}^{\infty} \mathrm{d} v_{x} \int_{0}^{\infty} \mathrm{d} v_{\perp} v_{\perp}^{2} \bar{f}_{ \pm 1 b}$.

The relevant harmonic coefficients $\bar{f}_{m b}, \quad(m= \pm 1,0)$, are determined by the perturbed Vlasov equation (the SNG model is always assumed to have "external" sources and sinks),

$$
\begin{aligned}
\left(\partial_{t}+v_{x} \partial_{x}\right. & \left.-\Omega_{b} \partial_{\varphi}\right) f_{b} \\
& =-\frac{N_{b} q_{b}}{m_{b}}(\mathbf{E}+\mathbf{v} \times \mathbf{B}) \cdot \frac{\partial\left[G_{o b}\left(v_{x}, v_{\perp}\right) \Phi(\Delta)\right]}{\partial \mathbf{v}},
\end{aligned}
$$

that, when transformed (in time and space), becomes

$$
\begin{aligned}
{\left[-i\left(\omega-k v_{x}\right)-\right.} & \left.\Omega_{b} \partial_{\varphi}\right] \bar{f}_{b} \\
= & -\frac{N_{b} q_{b}}{m_{b}} \sum_{n=-\infty}^{\infty}\left\{\left[\overline{\mathbf{E}}\left(\bar{\omega}_{-n}, k\right)\right.\right. \\
& \left.\left.+\mathbf{v} \times \overline{\mathbf{B}}\left(\bar{\omega}_{-n}, k\right)\right] \cdot \frac{\partial}{\partial \mathbf{v}}\left[G_{o b}\left(v_{x}, v_{\perp}\right) \phi_{n}\right]\right\}
\end{aligned}
$$

and determines

$$
\begin{aligned}
\bar{f}_{\mp 1 b}= & -i \frac{q_{b} N_{b}}{m_{b}\left(\omega \pm \Omega_{b}-k v_{x}\right)} \\
& \times\left\{\bar{E}_{x}\left(\hat{\omega}_{1}, k\right)\left[\frac{\partial G_{o b}}{\partial v_{x}}\right] \phi_{\mp 1} .\right. \\
& +\bar{E}_{\mp}(\omega, k)\left[\frac{k v_{\perp}}{\omega} \frac{\partial G_{o b}}{\partial v_{x}}+\left(1-\frac{k v_{x}}{\omega} \frac{\partial G_{o b}}{\partial v_{\perp}}\right)\right] \phi_{o} \\
& +\bar{E}_{ \pm}\left(\hat{\omega}_{2}, k\right)\left[\frac{k v_{\perp}}{\hat{\omega}_{2}} \frac{\partial G_{o b}}{\partial v_{x}}+\left(1-\frac{k v_{x}}{\hat{\omega}_{2}}\right)\right. \\
& \left.\left.\times\left(\frac{\partial G_{o b}}{\partial v_{\perp}}+2 \frac{G_{o b}}{v_{\perp}}\right)\right] \phi_{\mp 2}\right\} \\
\bar{f}_{o b}= & -i \frac{q_{b} N_{b}}{m_{b}\left(\omega-k v_{x}\right)} \\
& \times\left\{\bar{E}_{x}(\omega, k)\left[\frac{\partial G_{o b}}{\partial v_{x}}\right] \phi_{o}\right. \\
& +\bar{E}_{-}\left(\bar{\omega}_{-1}, k\right)\left[\frac{k v_{\perp}}{\bar{\omega}_{-1}} \frac{\partial G_{o b}}{\partial v_{x}}+\left(1-\frac{k v_{x}}{\bar{\omega}_{-1}}\right)\right. \\
& \left.\times\left(\frac{\partial G_{o b}}{\partial v_{\perp}}+\frac{G_{o b}}{v_{\perp}}\right)\right] \phi_{1} \\
& +\bar{E}_{+}\left(\bar{\omega}_{1}, k\right)\left[\frac{k v_{\perp}}{\bar{\omega}_{1}} \frac{\partial G_{o b}}{\partial v_{x}}+\left(1-\frac{k v_{x}}{\bar{\omega}_{1}}\right)\right. \\
& \left.\left.\times\left(\frac{\partial G_{o b}}{\partial v_{\perp}}+\frac{G_{o b}}{v_{\perp}}\right)\right] \phi_{-1}\right\} \\
& \left.=\omega_{0}\right\}
\end{aligned}
$$

with $\bar{\omega}_{n}=\omega+n \Omega_{b}\left(\bar{\omega}_{n}=\omega\right)$ and $\hat{\omega}_{n}=\omega \pm n \Omega_{b}\left(\hat{\omega}_{n}=\omega\right)$ for TNG (SNG) media. These results show that only the first two harmonics of the gyrophase distribution can influence the linear characteristics of parallel propagation in the nongyrotropic medium.

Two issues require clarification at this stage: (i) the differences between the TNG and SNG (with "external" sources and sinks) models and (ii) the nongyrotropic causes of mode coupling. With respect to the former, we note that whereas the spectral component $(\omega, k)$ of $\bar{f}_{b}$ for TNG media $\left(\Delta=\varphi+\Omega_{b} t\right)$ arises from the sum of the interactions of the $n$th harmonic of $\Phi, \phi_{n} \exp (-i n \Delta)$, with the wave electric and magnetic field spectral components $\left(\omega-n \boldsymbol{\Omega}_{b}, k\right)$, in SNG plasmas $(\Delta=\varphi)$ the relevant wave fields have the same spectral location $(\omega, k)$. This basic difference between TNG and SNG models is responsible for the occurrence of frequency shifted field components in the TNG (and not in the $\mathrm{SNG}$ ) matrix wave equation.

As to the roots of the interactions among the characteristic waves, both in the TNG and SNG cases, the above expressions explain the nongyrotropic mode coupling. For example, the generation of $\bar{f}_{\mp 1 b}$ (and hence of transverse electromagnetic circularly polarized right [-], or left [+] waves) can arise from: (i) transverse wave perturbations with the same $\left[\bar{E}_{\mp}\right]$ polarization acting on the gyrotropic component $\phi_{o}$ of the gyrophase distribution (uninteresting mechanism, already occurring in gyrotropic media), (ii) longitudinal electrostatic wave perturbations $\left[\bar{E}_{x}\right]$ acting on the $\phi_{\mp 1}$ harmonics of $\Phi$, and (iii) transverse wave perturbations with orthogonal polarizations $\left[\bar{E}_{ \pm}\right]$acting on the $\phi_{\mp 2}$ harmonics of $\Phi$. Recalling the rotating interpretation of the gyrophase 
harmonics (of $\bar{f}_{b}$ ), it is clear that mechanism (iii) is simply a stroboscopic effect in the perpendicular plane, whereas the interaction between longitudinal and transverse fields associated with (ii) is made possible, as explained elsewhere (e.g. Brinca, 1996), by the occurrence of an unperturbed finite perpendicular current density, $\overline{\mathbf{J}}_{b o} \neq 0$ (corresponding to $\phi_{ \pm 1} \neq 0$ ), that allows the density perturbation originated in the electrostatic longitudinal wave perturbation to stimulate a perturbed transverse current density.

The resulting wave and dispersion equations for parallel propagation are provided in Appendix A. It becomes clear that the nongyrotropic species (more exactly, the first two harmonics of its gyrophase distribution) is responsible for the coupling among the characteristic modes of parallel propagation, bringing about the nondiagonal elements of $m_{r s}$ in the wave matrix equation. Removal of gyrophase bunching yields the dispersion equation $m_{++} m_{x x} m--=0$ that defines the uncoupled gyrotropic dispersions of the left $(+)$, electrostatic (x) and right $(+)$ modes at the frequencies $\bar{\omega}_{1}, \omega$, and $\bar{\omega}_{-1}$, respectively. The wave and matrix equations are simpler for the SNG media. In the TNG case the spectral field components display the $\pm \Omega_{b}$ frequency shifts originated in the time-varying nongyrotropic distribution, and the matrix elements $m_{r s}$ are more complicated. However, the mechanisms underlying the coupling of the eigenmodes of parallel propagation are identical in both models.

As already stated, the investigations concerned with SNG magnetoplasmas have assumed that the source and sink terms of the Vlasov equation for the nongyrotropic species are external to the system $\left(S_{1}=L_{1}=0\right)$. Less drastic hypotheses can be envisaged. Cao et al. (1995) and Motschmann et al. (1997) have studied SNG environments arising from source and loss terms satisfying $S=S_{o}\left(S_{1}=0\right)$ and $L=F_{b} / \tau\left(L_{1}=F_{b 1} / \tau\right)$; they have failed to consider, however, the influence of $L_{1}$ (through the parameter $\tau$ ) on the dispersion, and hence stability, of the nongyrotropic system.

\subsection{Perpendicular propagation}

Even in gyrotropic media, the dispersion of oblique (perpendicular, in particular) propagation, when compared to the parallel case, is considerably more complicated. Introduction of species with gyrophase organization compounds the problem and brings about dispersion equations for nongyrotropic warm magnetoplasmas that, in general, can only be (numerically) solved with the adoption of extreme simplifications.

In contrast to the nongyrotropic parallel dispersion where only the first two harmonics of the gyrophase distribution influence the behavior of the system, at oblique propagation all the harmonics can integrate the dispersion equation. Whereas in parallel propagation there exist, at most, three interacting modes with (TNG), or without (SNG) frequency shifts in their spectral field components, oblique propagation in TNG plasmas couples all the frequency shifted $\left(\omega-n \Omega_{b}\right)$ field components yielding a dispersion equation that results from the annulment of an infinite order determinant whose elements contain infinite sums. The study of TNG characteristics under these circumstances is better achieved through appropriate numerical simulations. Oblique dispersion in SNG plasmas is somewhat simpler and, as described below, even amenable to full analytical treatment in special cases.

The difficulties arising from gyrophase bunching can be traced to the transformed structure of the perturbed current density associated with the nongyrotropic species in oblique propagation, $\overline{\mathbf{J}}_{b}(\omega, \mathbf{k})$. In general, all the harmonics of the gyrophase distribution contribute to the elements of the generalized conductivity tensor; in SNG media, this current density spectral component is stimulated by similar spectral components of the wave fields (allowing for a conductivity tensor of order 3), whereas for TNG plasmas $\overline{\mathbf{J}}_{b}(\omega, \mathbf{k})$ results from the contributions of all the frequency shifted spectral components $\left(\omega-n \Omega_{b}, \mathbf{k}\right)$ of the wave fields (generating an infinite order conductivity tensor).

Investigations of perpendicular propagation in TNG magnetoplasmas started with Eldridge (1970) who derived the electrostatic dispersion equation and discussed its solutions for very weak nongyrotropies. Brinca et al. (1994, 1998) reported (preliminary and comprehensive) results of the first numerical simulations of perpendicular propagation in TNG media. Cao et al. (1998) looked at oblique propagation in cold SNG (external sources and sinks) media, and Motschmann and Glaßmeier (1998) discussed the oblique dispersion in SNG (external sources and sinks) and TNG environments albeit presenting numerical (perpendicular propagation) solutions only for the cold plasma approximation. Romeiras and Brinca (unpublished) derived the dispersion equation satisfied by perpendicular electrostatic waves in SNG media (external sources and sinks) with monochromatic or dichromatic gyrophase distributions and demonstrated analytically the occurrence of a strong instability (growth rates of the order of the real frequencies) for large wavelengths (as compared to the thermal gyroradii) below a certain critical value; full numerical solution of the dispersion equation may uncover other instability regimes.

\section{Effects of nongyrotropy}

\subsection{Parallel propagation}

It has been stressed that the introduction of gyrophase bunching in a particle species brings about coupling among the characteristic modes of parallel propagation. These interactions depend on the values of the Fourier coefficients of the first two harmonics of the gyrophase distribution: other conditions being identical, a coarse assessment of the intensity of the nongyrotropic effects can be made from the magnitudes of these Fourier coefficients. Coupling between the electrostatic and electromagnetic modes requires the existence of a finite first harmonic $\left(\phi_{1}=\left(\phi_{-1}\right)^{*} \neq 0\right)$, whereas the left and 
right electromagnetic modes interact when there exists a second harmonic $\left(\phi_{2}=\left(\phi_{-2}\right)^{*} \neq 0\right)$; the remaining harmonics of the gyrophase distribution do not influence the linear behavior of parallel propagation. Satisfaction of $\phi_{1}=\left(\phi_{-1}\right)^{*} \neq 0$ is tantamount to the occurrence of a finite unperturbed perpendicular current density, $\mathbf{J}_{b o} \neq 0$, that generates an additional magnetic field and thus, strictly speaking, invalidates the assumed unperturbed state. The (ir)relevance of this effect to the ensuing analysis is discussed elsewhere (e.g. Brinca and Romeiras, 1998). For the sake of systematizing, the distinction between unperturbed states with (unbalanced), or without (balanced) finite perpendicular current densities shall be added to the differenciation between TNG and SNG magnetoplasmas.

The studies of parallel propagation in TNG media have solved the appropriate dispersion relation (Brinca et al., 1992, 1993a, 1993b; Motschmann and Glaßmeier, 1993) and used numerical simulations with hybrid (Brinca et al., 1993a, Motschmann et al., 1997) or kinetic (Brinca et al., 1993b) codes. They showed that time-varying gyrophase bunching can (i) enhance preexisting gyrotropic instabilities and (ii) destabilize otherwise thoroughly passive magnetoplasmas, albeit the later effect (ii) requires the existence of unbalanced gyrophase organization $\left(\phi_{1}=\left(\phi_{-1}\right)^{*} \neq 0, \mathbf{J}_{b o} \neq 0\right)$. The numerical simulations confirmed the linear characteristics determined by the dispersion equation (bearing in mind the frequency shifts brought about by the timevarying unperturbed state and their appropriate interpretation (e.g. Brinca, 1996) and evidenced that the nonlinear evolution of the system is accompanied by diffusion in velocity space that gradually smears the initial gyrophase bunching.

To date, investigations of parallel stability in SNG plasmas (Cao et al., 1995, Motschmann et al., 1997; Brinca and Romeiras, 1998; Motschmann and Glaßmeier, 1998) have always (implicitly or explicitly) assumed that the necessary source and, or, sink terms in the Vlasov equation satisfied by the distribution of the nongyrotropic species were "external" to the system, that is, those terms did not influence the small perturbations of the medium and thus did not affect its linear dispersion; future work in this area should incorporate, when appropriate, the effects of "internal" sources and, or, sinks in the analysis. The study of the solutions of the SNG parallel dispersion equation under these circumstances ("external" sources and sinks) has shown that the stationary gyrophase bunching can also, as in the TNG case, (i) enhance pre-existing gyrotropic instabilities and (ii) destabilize otherwise passive media. However, in the SNG gyrophase bunching and in contrast to the TNG case, the destabilizing effect (ii) also occurs for balanced distributions $\left(\phi_{1}=\left(\phi_{-1}\right)^{*}=\mathbf{J}_{b o}=0\right)$, meaning that the interaction between the two parallel electromagnetic modes (originated in $\phi_{2}=\left(\phi_{-2}\right)^{*} \neq 0$ ) can be sufficient to bring about wave growth in an otherwise stable magnetoplasma; under extreme conditions (Brinca and Romeiras, 1998), stationary balanced gyrophase organization can stimulate nonoscillatory pure growth (positive growth rates with zero real frequencies within a finite range of wavenumbers). Simulation results for stationary gyrophase organization are not available: the eventual particle recycling necessary to generate SNG seems too intense to provide reliable results.

\subsection{Perpendicular propagation}

The analytical study of oblique propagation in nongyrotropic media is unwieldy. When requiring nontrivial solutions for the wave matrix equation to obtain the dispersion equation, the associated determinant (whose annulment is imposed) is of order 3, as in gyrotropic oblique propagation, for SNG plasmas and of infinite order for the TNG case. In both situations the elements of the determinants depend, in general, on all the Fourier coefficients of the gyrophase distribution. Not surprisingly, studies of oblique (perpendicular, in particular) propagation in nongyrotropic magnetoplasmas supported in the (analytical or numerical) solution of the appropriate dispersion equation are scarce, adopt simplifying models, and usually relate to SNG environments with external sources and sinks.

Motschmann and Glaßmeier (1998) investigated the oblique dispersion in SNG (external sources and sinks) and TNG media; the numerical solutions for perpendicular propagation envisage a magnetoplasma with gyrotropic electrons and protons permeated by unbalanced gyrophase bunched alpha particles, in the cold plasma approximation. They obtain extraordinarymode instabilities stimulated by the nongyrotropic species, albeit in the TNG model the parameters fall outside the range of validity of the adopted approximation. Perpendicular electrostatic propagation in SNG (external sources and sinks) media with extreme (monochromatic or dichromatic) gyrophase organization satisfies a reasonably simple dispersion equation (Romeiras and Brinca, unpublished) that, when analytically solved for large (with respect to the thermal gyroradii) wavenumbers, demonstrates that the SNG free energy can feed strong perpendicular electrostatic wave growth; other instabilities may be found through the full numerical solution of the derived dispersion equation.

Numerical simulations are usually adopted to study perpendicular propagation in TNG environments (Brinca et al., 1994, 1998). They can easily assess the influence of more realistic gyrophase distributions and provide information on the nonlinear evolution of the encountered instabilities. The results obtained for perpendicular electrostatic stability show that both balanced and unbalanced time-varying nongyrotropies can destabilize an otherwise passive medium. Use of an electromagnetic code shows that this electrostatic instability may coexist with the growth of the extraordinary mode (the ordinary mode was found to be stable). Sensibly, the evolution towards saturation is accompanied by a gradual gyrotropization of the initial gyrophase bunching; luckily, wave growth is reasonably resilient with respect to the degree of gyrophase organization. It is interesting to notice that, for parallel propagation, the capacity of 
TNG environments to destabilize otherwise passive media could only be found in unbalanced gyrophase distributions.

\section{Discussion}

Following a discussion of the physics implicated in the interactions brought about by the introduction of gyrophase bunching in homogeneous magnetoplasmas, and as a prologue to future investigations on the characteristics of homogeneous nongyrotropic environments, the available stability results were qualitatively described for parallel and perpendicular propagation, TNG and SNG (external sources and sinks) media. Both analytic and simulation studies evidenced the proclivity of nongyrotropy towards instability.

Space observations have demonstrated that nongyrotropic particle populations are frequently encountered in the geoplasma. Theoretical studies have amply proved that the free energy associated with gyrophase organization can enhance pre-existing gyrotropic instabilities and destabilize otherwise passive media. A solid connection between these two undisputed assertions remains to be made in order to assess the relevance of nongyrotropy to space plasma phenomenology. In particular, the full characterization of the observed nongyrotropic distributions and adequate modeling of the associated sources and sinks are necessary to improve the theoretical approaches and adapt them to natural environments. Potential applications to concrete space physics problems shall then follow and are plentiful. Leaving aside the (very important, but not discussed here) implications of inhomogeneous gyrophase bunchings, as occurring for instance in (Harris type) current-sheet models found in the magnetopause and magnetotail (viz. possible relevance to reconnection (Motschmann and Glaßmeier, 1997)), the (re)interpretation of wave activity coexisting with observed (or suspected) nongyrotropic species is warranted. Two examples: wave fields in the neighborhood of the space shuttle (e.g. Rivas and Hastings, 1992) and intense bursts of electron cyclotron harmonic emissions near the dayside magnetopause (Matsumoto and Usui, 1997) that cannot be explained by classical gyrotropic instabilities.

Acknowledgments. Fruitful discussions with the members of the Nongyrotropy in Space Plasmas project are gratefully acknowledged. This work was partially supported by FEDER and PRAXIS XXI.

Topical Editor K.-H. Glaßmeier thanks C. Mazelle and A. Coates for their help in evaluating this paper.

\section{Appendix}

\section{Wave and dispersion equations for parallel propagation}

The resulting wave and dispersion equations for the initial value problem with field quantities proportional to $\exp i(k x-\omega t)$ shows that the introduction of gyro- phase organization in the magnetoplasma brings about, in general, coupling among the eigenmodes of the system (left- $[+]$ and right-hand [-] circularly polarized electromagnetic and longitudinal electrostatic $[x]$ waves).

The matrix wave equation assumes the form

$$
\left(\begin{array}{ccc}
m_{++} & m_{+x} & m_{+-} \\
m_{x+} & m_{x x} & m_{x-} \\
m_{-+} & m_{-x} & m_{--}
\end{array}\right)\left(\begin{array}{c}
\bar{E}_{+}\left(\bar{\omega}_{1}, k\right) \\
\bar{E}_{x}(\omega, k) \\
\bar{E}_{-}\left(\bar{\omega}_{-1}, k\right)
\end{array}\right)=0,
$$

with

$$
\begin{aligned}
& m_{++}=\left(\frac{k c}{\bar{\omega}_{1}}\right)^{2}-1-\frac{\pi}{\bar{\omega}_{1}} \\
& \times\left\{\sum_{l=e, p} \omega_{p l}^{2} \int_{-\infty}^{\infty} \frac{\mathrm{d} v_{x}}{\bar{\omega}_{1}-k v_{x}-\Omega_{l}}\right. \\
& \times \int_{0}^{\infty} \mathrm{d} v_{\perp} v_{\perp}^{2}\left[\left(1-\frac{k v_{x}}{\bar{\omega}_{1}}\right) \frac{\partial F_{o l}}{\partial v_{\perp}}+\frac{k v_{\perp}}{\bar{\omega}_{1}} \frac{\partial F_{o l}}{\partial v_{x}}\right] \\
& +\omega_{p b}^{2} \int_{-\infty}^{\infty} \frac{\mathrm{d} v_{x}}{\omega-k v_{x}} \int_{0}^{\infty} \mathrm{d} v_{\perp} v_{\perp}^{2} \\
& \left.\times\left[\left(1-\frac{k v_{x}}{\bar{\omega}_{1}}\right) \frac{\partial G_{o b}}{\partial v_{\perp}}+\frac{k v_{\perp}}{\bar{\omega}_{1}} \frac{\partial G_{o b}}{\partial v_{x}}\right] \phi_{o}\right\} \\
& m_{+x}=-\frac{\pi}{\bar{\omega}_{1}} \omega_{p b}^{2} \int_{-\infty}^{\infty} \frac{\mathrm{d} v_{x}}{\omega-k v_{x}} \int_{0}^{\infty} \mathrm{d} v_{\perp} v_{\perp}^{2}\left[\frac{\partial G_{o b}}{\partial v_{x}}\right] \phi_{1} \\
& m_{+-}=-\frac{\pi}{\bar{\omega}_{1}} \omega_{p b}^{2} \int_{-\infty}^{\infty} \frac{\mathrm{d} v_{x}}{\omega-k v_{x}} \int_{0}^{\infty} \mathrm{d} v_{\perp} v_{\perp}^{2}\left[\frac{k v_{\perp}}{\bar{\omega}_{-1}} \frac{\partial G_{o b}}{\partial v_{x}}\right] \phi_{2} \\
& m_{x+}=\frac{2 \pi}{\omega} \omega_{p b}^{2} \int_{-\infty}^{\infty} \mathrm{d} v_{x} \frac{v_{x}}{\omega-k v_{x}} \int_{0}^{\infty} \mathrm{d} v_{\perp} v_{\perp}\left[\frac{k v_{\perp}}{\bar{\omega}_{1}} \frac{\partial G_{o b}}{\partial v_{x}}\right] \phi_{-1} \\
& m_{x x}=1+\frac{2 \pi}{\omega}\left\{\sum_{l=e, p} \omega_{p l}^{2} \int_{-\infty}^{\infty} \mathrm{d} v_{x} \frac{v_{x}}{\omega-k v_{x}}\right. \\
& \times \int_{0}^{\infty} \mathrm{d} v_{\perp} v_{\perp}\left[\frac{\partial F_{o l}}{\partial v_{x}}\right]+\omega_{p b}^{2} \int_{-\infty}^{\infty} \mathrm{d} v_{x} \frac{v_{x}}{\omega-k v_{x}} \\
& \left.\times \int_{0}^{\infty} \mathrm{d} v_{\perp} v_{\perp}\left[\frac{\partial G_{o b}}{\partial v_{x}}\right] \phi_{o}\right\} \\
& m_{x-}=\frac{2 \pi}{\omega} \omega_{p b}^{2} \int_{-\infty}^{\infty} \frac{\mathrm{d} v_{x} v_{x}}{\omega-k v_{x}} \int_{0}^{\infty} \mathrm{d} v_{\perp} v_{\perp}\left[\frac{k v_{\perp}}{\bar{\omega}_{-1}} \frac{\partial G_{o b}}{\partial v_{x}}\right] \phi_{1} \\
& m_{--}=-\frac{\pi}{\bar{\omega}_{-1}} \omega_{p b}^{2} \int_{-\infty}^{\infty} \frac{\mathrm{d} v_{x}}{\omega-k v_{x}} \int_{0}^{\infty} \mathrm{d} v_{\perp} v_{\perp}^{2}\left[\frac{k v_{\perp}}{\bar{\omega}_{1}} \frac{\partial G_{o b}}{\partial v_{x}}\right] \phi_{-2}
\end{aligned}
$$




$$
\begin{aligned}
m_{-x}= & -\frac{\pi}{\bar{\omega}_{-1}} \omega_{p b}^{2} \int_{-\infty}^{\infty} \frac{\mathrm{d} v_{x}}{\omega-k v_{x}} \int_{0}^{\infty} \mathrm{d} v_{\perp} v_{\perp}^{2}\left[\frac{\partial G_{o b}}{\partial v_{x}}\right] \phi_{-1} \\
m_{--}= & \left(\frac{k c}{\bar{\omega}-1}\right)^{2}-1-\frac{\pi}{\bar{\omega}_{-1}} \\
& \times\left\{\sum_{l=e, p} \omega_{p l}^{2} \int_{-\infty}^{\infty} \frac{\mathrm{d} v_{x}}{\bar{\omega}_{-1}-k v_{x}+\Omega_{l}}\right. \\
& \times \int_{0}^{\infty} \mathrm{d} v_{\perp} v_{\perp}^{2}\left[\left(1-\frac{k v_{x}}{\bar{\omega}_{-1}}\right) \frac{\partial F_{o l}}{\partial v_{\perp}}+\frac{k v_{\perp}}{\bar{\omega}_{-1}} \frac{\partial F_{o l}}{\partial v_{x}}\right] \\
& +\omega_{p b}^{2} \int_{-\infty}^{\infty} \frac{\mathrm{d} v_{x}}{\omega-k v_{x}} \int_{0}^{\infty} \mathrm{d} v_{\perp} v_{\perp}^{2} \\
& \left.\times\left[\left(1-\frac{k v_{x}}{\bar{\omega}}\right) \frac{\partial G_{-1}}{\partial v_{\perp}}+\frac{k v_{\perp}}{\bar{\omega}_{-1}} \frac{\partial G_{o b}}{\partial v_{x}}\right] \phi_{o}\right\}
\end{aligned}
$$

where we recall that $\bar{\omega}_{ \pm 1}=\omega \pm \Omega_{b}\left(\bar{\omega}_{ \pm 1}=\omega\right)$ for TNG (SNG) magnetoplasmas. The spectral electric field components in the matrix wave equation for the TNG model are frequency shifted by $+\Omega_{b}$ with respect to results presented elsewhere (e.g. Brinca et al., 1993a) and similar shifts occur in the expressions of the matrix elements $m_{r s}$. Both versions are correct and the interpretation of the associated dispersion is given in Brinca (1996).

When the particle species have (possibly drifting and anisotropic) Maxwellian distributions, that is,

$$
\begin{aligned}
F_{o l} & =M_{l}\left(v_{t l}, V_{D l}, A_{l}\right) \\
& =\frac{1}{A_{l}\left(\sqrt{\pi} v_{t l}\right)^{3}} \mathrm{e}^{-\left(v_{x}-V_{D l}\right)^{2} / v_{t l}{ }^{2}} \mathrm{e}^{-v_{\perp}{ }^{2} /\left(A_{l} v_{t l}{ }^{2}\right)}
\end{aligned}
$$

for the gyrotropic populations $(l=e, p)$, and

$F_{o b}=G_{o b}\left(v_{x}, v_{\perp}\right) \Phi_{b}(\Delta)=2 \pi M_{b}\left(v_{t b}, V_{D b}, A_{b}\right) \Phi_{b}(\Delta)$,

for the nongyrotropic particles, with anisotropies, parallel drifts and thermal velocities denoted by $A_{s}=T_{\perp s} / T_{x s}, \quad V_{D s}$, and $v_{t s}=\left(2 T_{x s / m_{s}}\right)^{1 / 2}$ where $s=e$, $p, b$, the matrix elements $m_{r s}$ become

$$
\begin{aligned}
m_{++}= & \left(\frac{k c}{\bar{\omega}_{1}}\right)^{2}-1-\sum_{s=e, p, b}\left(\frac{\omega_{p s}}{\bar{\omega}_{1}}\right)^{2} \\
& \times\left[\frac{\bar{\omega}_{1}}{k v_{t s}}\left(1-\frac{k V_{D s}}{\bar{\omega}_{1}}\right) Z\left(\xi_{s+}\right)-\frac{1}{2}\left(A_{s}-1\right) Z^{\prime}\left(\xi_{s+}\right)\right] \\
m_{+x}= & \frac{\pi^{3 / 2}}{2} \sqrt{A_{b}}\left(\frac{\omega_{p b}}{\bar{\omega}_{1}}\right)^{2} \frac{\bar{\omega}_{1}}{k v_{t b}} \phi_{1} Z^{\prime}\left(\xi_{b+}\right) \\
m_{+-}= & \pi A_{b} \frac{\omega_{p b}^{2}}{\bar{\omega}_{-1} \bar{\omega}_{1}} \phi_{2} Z^{\prime}\left(\xi_{b+}\right) \\
m_{x+}= & -\pi^{3 / 2} \sqrt{A_{b}} \frac{\omega_{p b}^{2}}{\bar{\omega}_{1} k v_{t b}} \phi_{-1} Z^{\prime}\left(\xi_{b x}\right)
\end{aligned}
$$

$$
\begin{aligned}
m_{x x}= & 1-\sum_{s=e, p, b}\left(\frac{\omega_{p s}}{k v_{t s}}\right)^{2} Z^{\prime}\left(\xi_{s x}\right) \\
m_{x-}= & -\pi^{3 / 2} \sqrt{A_{b}} \frac{\omega_{p b}^{2}}{\bar{\omega}_{-1} k v_{t b}} \phi_{1} Z^{\prime}\left(\xi_{b x}\right) \\
m_{-+}= & \pi A_{b} \frac{\omega_{p b}^{2}}{\bar{\omega}_{-1} \bar{\omega}_{1}} \phi_{-2} Z^{\prime}\left(\xi_{b-}\right) \\
m_{-x}= & \frac{\pi^{3 / 2}}{2} \sqrt{A_{b}}\left(\frac{\omega_{p b}}{\bar{\omega}_{-1}}\right)^{2} \frac{\bar{\omega}_{-1}}{k v_{t b}} \phi_{-1} Z^{\prime}\left(\xi_{b-}\right) \\
m_{--}= & \left(\frac{k c}{\bar{\omega}_{-1}}\right)^{2}-1-\sum_{s=e, p, b}\left(\frac{\omega_{p s}}{\bar{\omega}_{-1}}\right)^{2} \\
& \times\left[\frac{\bar{\omega}_{-1}}{k v_{t s}}\left(1-\frac{k V_{D s}}{\bar{\omega}_{-1}}\right) Z\left(\xi_{s-}\right)-\frac{1}{2}\left(A_{s}-1\right) Z^{\prime}\left(\xi_{s-}\right)\right]
\end{aligned}
$$

where

$$
\xi_{s \pm}=\frac{\bar{\omega}_{ \pm 1}-k V_{D j} \mp \Omega_{s}}{k v_{t s}} \quad \xi_{s x}=\frac{\omega-k V_{D s}}{k v_{t s}}
$$

and $Z(\xi)$ and $Z^{\prime}(\xi)$ represent the plasma dispersion function and its derivative.

The dispersion equation allows for the occurrence of nontrivial solutions and is defined by

$\operatorname{det}\left(m_{r s}\right)=D\left(\omega=\omega_{r}+i \omega_{i}, k\right)=0$.

\section{References}

Borovski, J. E., Properties and dynamics of the electron beams emanating from magnetized plasma double layers, J. Geophys. Res., 93, 5713-5725, 1988.

Brinca, A. L., Observation and interpretation of cometary low frequency waves, Surv Geophys., 17, 1-39, 1996.

Brinca, A. L., and F. J. Romeiras, On the stability of stationary nongyrotropic distribution functions: coupling and purely growing waves, J. Geophys. Res., 103, 9275-9284, 1998

Brinca, A. L., L. Borda de Água, and D. Winske, Nongyrotropy as a source of instability and mode coupling, Geophys. Res. Lett., 19, 2445-2448, 1992.

Brinca, A. L., L. Borda de Água, and D. Winske, On the stability of nongyrotropic ion populations: a first (analytic and simulation) assessment, J. Geophys. Res., 98, 7549-7560, 1993a.

Brinca, A. L., Y. Omura, and H. Matsumoto, Linear coupling effects originated in electron nongyrotropy, J. Geophys. Res., 98, 21071-21076, 1993b.

Brinca, A. L., Y. Omura, and H. Matsumoto, Bernstein modes in nongyrotropic space plasmas, International Conference on Plasma Physics, Foz do Iguaçu (Brazil), Oct. 31-Nov 4, 1994.

Brinca, A. L., Y. Omura, and H. Matsumoto, Stability of perpendicular propagation in time-varying nongyrotropic plasmas: Simulations, J. Geophys. Res. 103, 29493-29503, 1998.

Burgess, D., Simulations of backstreaming ion beams formed at oblique shocks by direct reflection, Ann. Geophysicae., 5, 133145, 1987.

Cao, J. B., C. Mazelle, G. Belmont, and H. Reme, Nongyrotropy of heavy newborn ions at comet Grigg-Skjellerup and corresponding instability, J. Geophys. Res., 100, 23379-23388, 1995.

Cao, J. B., C. Mazelle, G. Belmont, and H. Reme, Oblique ring instability driven by nongyrotropic ions: Application to observations at comet Grigg-Skjellerup, J. Geophys. Res., 103, 20552067, 1998. 
Coates, A. J., A. D. Johnstone, B. Wilken, and F. M. Neubauer, Velocity space diffusion and nongyrotropy of pickup water group ions at comet Grigg-Skjellerup, J. Geophys. Res., 98, 20985-20994, 1993.

Delcourt, D. C., J.-A. Sauvaud, and T. E. Moore, Phase bunching during substorm dipolarization, J. Geophys. Res., 102, 2431324324, 1997.

Eastman, T. E., R. R. Anderson, and L. A. Frank, Upstream particles observed in the Earth's foreshock region, J. Geophys. Res., 86, 4379, 1981.

Eldridge, O., Coupling of electrostatic waves in a nongyrotropic plasma, Phys. Fluids, 13, 1791-1794, 1970.

Gurgiolo, C., G. K. Parks, B. H. Mauk, C. S. Lin, K. A. Anderson, R. P. Lin, and H. Reme, Non-E $\times$ B ordered ion beams upstream of the Earth's bow shock, J. Geophys. Res., 86, 44154424, 1981.

Hoshino, M., and T. Terasawa, Numerical study of the upstream wave excitation mechanism, 1, Nonlinear phase bunching of beam ions, J. Geophys. Res., 90, 57-64, 1985.

Matsumoto, H., and H. Usui, Intense bursts of electron cyclotron harmonic waves near the dayside magnetopause observed by GEOTAIL, Geophys. Res. Lett., 24, 49-52, 1997.
Motschmann, U., and K.-H. Glaßmeier, Nongyrotropic distribution of pickup ions at comet P/G-S: A possible source of wave activity, J. Geophys. Res., 98, 20977-20983, 1993.

Motschmann, U., and K.-H. Glaßmeier, Concerning nongyrotropic particle distributions and magnetic field reconnection, AGU Fall Meeting, San Francisco, December 1997.

Motschmann, U., and K.-H. Glaßmeier, Dispersion and wave excitation in nongyrotropic plasmas, J. Plasma Phys., 60, 111132, 1998.

Motschmann, U., H. Kafemann, and M. Scholer, Nongyrotropy in magnetoplasmas: Simulation of wave excitation and phasespace diffusion, Ann. Geophysicae., 15, 603-613, 1997.

Neubauer, F. M., K.-H. Glaßmeier, A. J. Coates, and A. D. Johnstone, Low frequency electromagnetic plasma waves at comet P/Grigg-Skjellerup: Analysis and interpretation, J. Geophys. Res., 98, 20937-20953, 1993.

Rivas, D. R., and D. E. Hastings, Theoretical interpretation of the electrostatic waves in the space shuttle induced plasma environment, J. Geophys. Res., 97, 17097-17113, 1992.

Sudan, R. N., Growing waves in a nongyrotropic plasma, Phys. Fluids, 8, 1915-1918, 1965. 